This is a postprint of Fluid phase equilibria, 231(2), 231-238. The original article can be found under http://www.sciencedirect.com/science/article/pii/S037838120500021X

\title{
Vapor-liquid equilibrium prediction at high pressures using activity coefficients at infinite dilution from COSMO-type methods
}

\author{
Dana Constantinescu ${ }^{\mathrm{a}}$, Andreas Klamt ${ }^{\mathrm{b}}$, Dan Geană ${ }^{\mathrm{a}, *}$ \\ a Dept. of Applied Physical Chemistry, University Polytechnica of Bucharest, Spl. Independentei 313, \\ Bucharest, Romania \\ b Cosmologic GmbH and Co. KG, Burscheider Str. 515, Leverkusen 51381, Germany
}

\begin{abstract}
Predictions of vapor-liquid equilibria at high temperatures and pressures were obtained by applying a modified procedure using the Huron-Vidal mixing rule based on available activity coefficients at infinite dilution and low pressures. These activity coefficients were calculated with so-called COSMO-RS (conductor-like screening model for real solvents) and with a thermodynamically variation of this model, known as COSMO-SAC (segment activity coefficient) model.

In this work, the performances of the mixing rule (HVID model) coupled with the SRK equation of state and a reduced UNIQUAC model are presented for six binary systems and a ternary system, whose VLE data are available over a large temperature and pressure range.
\end{abstract}

Keywords: method of calculation, vapor-liquid equilibria, equation of state, activity coefficient, COSMORS model, COSMO-SAC model.

\section{Introduction}

Infinite dilution activity coefficients $\left(\gamma^{\infty}\right)$ are of great importance in both chemical and environmental engineering. If both infinite dilution activity coefficients are known for a binary system, parameters in a two-parameter activity coefficient model can be determined and then predictions of vapor-liquid equilibria over the entire composition range can be made.

The use of infinite dilution activity coefficients at low pressure in cubic equations of state was discussed by Feroiu and Geană [1] in relation to the Stryjek and Vera [2] nonquadratic mixing rule. In previous papers [3-6], Feroiu and Geană proposed a modified procedure of using available activity coefficients at infinite dilution in the Huron-Vidal mixing rule (HVID). Following previous work [5,6], in which the activity coefficients at infinite dilution were calculated with one of the most successful predictive $\mathrm{G}^{\mathrm{E}}$ models, i.e. UNIFAC'93 [7], we suggest here to make use of a novel method for the prediction of thermophysical data of liquids. This method, called 'Conductor like Screening Model for Real Solvents' (COSMO-RS) was originally published in 1995 [8].

\footnotetext{
* to whom the correspondence should be addressed

E-mail address: d_geana @ catedra.chfiz.pub.ro;
} 
In contrast to the widely used group contribution methods, COSMO-RS gets the necessary information about the intermolecular interactions in solution from quantum mechanical calculations on the chemical compounds and thus is far less dependent on experimental data [9]. In a series of publications [9-12], it was shown that COSMO-RS is a valuable tool for handling of chemical and engineering thermodynamics problems concerning activity coefficients. In addition, it provides a great flexibility in treating systems of different chemical functionality. In a more recent paper [13] infinite dilution activity coefficients were predicted using the COSMO-RS model for over 400 binary systems.

Lin and Sandler [14] proposed a derivation leading to an activity coefficient model called COSMO-SAC based on their previously developed group contribution solvation model GCS [15].

The application of the COSMO models in making a priori phase equilibrium predictions at low pressures was reported by Spuhl and Arlt [16]. The limitations of the COSMO method are, at present, on one hand its inability to account for high-temperature and highpressure vapor-liquid equilibria, and on the other hand its inability to properly account for the thermodynamics of polymer systems. Panayiotou [17] incorporated free volume in the formalism and derived an equation of state that is applicable to polymer solutions.

The scope of this paper is to enlarge the fields of application of COSMO type methods to vapor-liquid equilibrium prediction at high pressures within the context of the proposed HVID model [3-6].

\section{Calculation model}

In this work, the cubic equation of state of Soave, Redlich and Kwong, SRK [18] was used:

$$
P=\frac{R T}{V-b}-\frac{a}{V(V+b)}
$$

The parameters for pure substances are:

$$
a_{i}=0.42748 \frac{R^{2} T_{c i}^{2}}{P_{c i}}\left[f\left(T_{r}\right)\right]^{2} ; \quad b_{i}=0.08664 \frac{R T_{c i}}{P_{c i}}
$$

where the Mathias and Copeman [19] temperature function $f\left(T_{r}\right)$ is given by:

$$
f\left(T_{r}\right)=1+C_{1} y+C_{2} y^{2}+C_{3} y^{3} \quad \text { for } \quad T_{r} \leq 1
$$

and

$$
f\left(T_{r}\right)=1+C_{1} y \quad \text { for } \quad T_{r} \geq 1
$$

with

$$
y=1-\sqrt{T_{r}}
$$


The critical constants and the values of $\mathrm{C}_{1}, \mathrm{C}_{2}$, and $\mathrm{C}_{3}$ constants were taken from Reid et al. [20] and Dahl et al. [21] respectively.

For mixtures, the Huron-Vidal mixing rules were used:

$$
\begin{gathered}
b=\sum_{i} x_{i} b_{i} \\
\frac{a}{b R T}=\sum_{i} x_{i}\left(\frac{a_{i}}{b_{i} R T}-\frac{\ln \gamma_{i}(P \rightarrow \infty)}{\ln 2}\right)
\end{gathered}
$$

where $\ln \gamma_{i}(P \rightarrow \infty)$ is the activity coefficient of the component in the mixture at infinite pressure.

The corresponding expression for the activity coefficient, which relates the infinite dilution activity coefficient at infinite pressure $\ln \gamma_{i(j)}^{\infty}(P \rightarrow \infty)$ to its value at pressure $\mathrm{P}$ $\left(\gamma_{i(j)}^{\infty}\right)$, is:

$$
\ln \gamma_{i(j)}^{\infty}(P)=\frac{b_{i}}{b_{j}}\left(Z_{j}-1\right)-\ln \left(\frac{V_{j}-b_{j}}{V_{i}-b_{i}}\right)-\left[\frac{a_{i}}{b_{i} R T} \ln \left(\frac{1+b_{j} / V_{j}}{1+b_{i} / V_{i}}\right)+\frac{\ln \gamma_{i(j)}^{\infty}(P \rightarrow \infty)}{\ln 2}\right] \ln \left(1+\frac{b_{j}}{V_{j}}\right)
$$

Details about the equation (8) can be seen in ref. [3-5, 22]. Moreover, if eq. (8) is applied under the limiting condition $P \rightarrow 0$, it yields $[1,2,22]$ :

$\ln \gamma_{i(j)}^{\infty}(P \rightarrow 0)=\left(1-\frac{b_{i}}{b_{j}}+\ln \frac{b_{i}}{b_{j}}\right)-\ln \left(\frac{V_{j} / b_{j}-1}{V_{i} / b_{i}-1}\right)-\frac{a_{i}}{b_{i} R T} \ln \left(\frac{1+b_{j} / V_{j}}{1+b_{i} / V_{i}}\right)+\frac{\ln \gamma_{i(j)}^{\infty}(P \rightarrow \infty)}{\ln 2} \ln \left(1+\frac{b_{j}}{V_{j}}\right)$

where $\left(V_{i}, V_{j}\right)$ are the solutions of eq. (1) at $P \rightarrow 0$.

Feroiu and Geană $[3,5]$ proposed a procedure to predict vapor-liquid equilibria at high pressure using the above mixing rule model and the infinite dilution activity coefficients at low pressures.

The activity coefficients in eq. (7) are given by a reduced UNIQUAQ model (only to its residual part, with the original values of $q_{i}$ parameters) suitable for infinite pressure conditions:

$$
\begin{array}{r}
\ln \gamma_{i}(P \rightarrow \infty)=q_{i}\left[1-\ln \left(\sum_{i} \theta_{j} \tau_{i j}\right)-\sum_{j} \frac{\theta_{j} \tau_{i j}}{\sum_{k} \theta_{k} \tau_{k j}}\right] \\
\theta_{i}=\frac{q_{i} x_{i}}{\sum q_{i} x_{j}} \quad \tau_{i j}=\exp \left(-\frac{u_{i j}}{T}\right)
\end{array}
$$

The temperature dependence of the parameter $u_{i j}$ is expressed by: 


$$
u_{i j}=u_{i j}^{o}+u_{i j}^{1} \frac{1}{T}
$$

The procedure involves the following five steps:

1. Use the activity coefficient model (COSMO-RS, COSMO-SAC or UNIFAC'93) at low temperature/low pressure, to calculate the activity coefficient at infinite dilution $\gamma_{i(j)}^{\infty}(P \rightarrow 0)$ for several data sets.

2. Calculation of the corresponding values of $\ln \gamma_{i}(P \rightarrow \infty)$ with eq. (9).

3. Calculation of the binary interaction parameters of the reduced UNIQUAC model at all temperatures.

4. Obtaining the linear temperature dependence of the interaction parameters (eq.12)

5. Calculation of vapor-liquid equilibrium at high temperatures/pressures based on HVID mixing rule.

According to the first step of the procedure, three sources for obtaining the values of infinite dilution activity coefficients have been used. COSMO-RS and COSMO-SAC models belong to a new class of methods, which is universally applicable with similar accuracy for all mixture. COSMO-parameters are not specific regarding functional groups or molecule types. Due to the generic functional for the interaction energies, which is based on the information from quantum mechanical calculations and which does only require a few element-specific parameters, these models are applicable to almost all organic molecules (including even experimentally unavailable entities such as reactive intermediates). This is the main practical difference between COSMO-types models and the group contribution models [13]. A comprehensive comparison of methodology in UNIFAC, COSMO-RS and COSMO-SAC was carried out by Klamt [9] and Lin and Sandler [14].

Following the premises of the COSMO-RS concept, the ensemble of interacting molecules may be replaced by the corresponding ensemble of independent, pairwise interacting surface pieces and the screening charge density $\sigma$ is the only descriptor for determining the interaction energy [9]. It is known that in UNIFAC model, an isolated molecule is broken into functional groups. The UNIFAC model uses group frequencies (i.e. the number of each functional group contained in a molecule). The group contribution frequency is analogous to the $\sigma$ profiles in COSMO-RS and COSMO-SAC, which are computed from partitioning an ideally screened molecule into surface segments.

The group contribution frequency and $\sigma$ profile represent the unique characteristic of each molecule. The sum of these profiles, each weighted by the mole fraction of each component, produces the profile of a mixture. In the UNIFAC model, the group activity coefficient is computed using the mixture group frequencies and a predetermined set of energy parameters. In contrast, the COSMO-RS and COSMO-SAC models determine the chemical potential of charged surface segments or segment activity coefficient from the mixture $\sigma$ profile with segment interactions calculated from the charged density on each segment.

Finally, the chemical potential and the activity coefficients are obtained by summing the contributions from groups or segments $[9,14]$. In this work, only the values of infinite 
dilution activity coefficients predicted by COSMO-RS, COSMO-SAC and UNIFAC'93 were used.

The MHV2 [21] and Wong-Sandler mixing rules [23] were used in a previous paper [3], and their predictions were compared with the HVID method. As shown in Table 5 in [3], the HVID mixing rule was better in its pressure and vapor phase predictions than the MHV2 and Wong-Sandler mixing rules. Therefore, in this work we studied only the HVID mixing rules.

\section{Results and discussion}

Six mixtures (listed in Table 2), whose experimental VLE data are available over a large temperature and pressure range, were selected in order to test the COSMO-RS, COSMO-SAC and UNIFAC'93 in predicting the vapor-liquid equilibria over a large range of conditions.

A modified version of UNIFAC model (UNIFAC'93) has been also used in this work. Compared to the original UNIFAC method, the UNIFAC'93 provides a better description of the temperature dependence of the activity coefficients and a more reliable representation of the real behavior of phase equilibria in the dilute region [7].

COSMO-RS calculations were made with COSMOtherm-version C1.2 program using TURBOMOLE quantum mechanical program files. Lin and Sandler [14] program V1.0 coupled with DMOL3 quantum mechanical program files was used for COSMO-SAC calculations. TURBOMOLE and DMOL3 files for the components of mixtures were available in COSMOlogic data base.

As an example, in Table 1 the activity coefficients at infinite dilution at low pressure, predicted with COSMO-RS, COSMO-SAC and UNIFAC'93 models together with the

corresponding calculated values of $\gamma_{i}^{\infty}(P \rightarrow \infty)$ for the acetone + water system are presented. As can be observed, the values of infinite dilution activity coefficients at infinite pressure are higher than those at zero pressure. The COSMO predicted values for the acetone-water system are lower than those calculated by UNIFAC' 93.

First, we studied the capability of the HVID model, based on COSMO-RS, COSMOSAC and UNIFAC'93 activity coefficients at infinite dilution, to represent the low temperature data sets available in literature [24-27]. The errors in pressure and vapor phase composition of the HVID- COSMO-RS, COSMO-SAC and UNIFAC'93 model are given comparatively in Table 2. The errors are reasonable for UNIFAC'93. As can be seen, the results for COSMO-RS, COSMO-SAC are not as good as those calculated with UNIFAC'93. In some cases, for methanol + benzene and 2-propanol + water, COSMOSAC shows poor results. One example of VLE predictions for the methanol + water binary system at $\mathrm{T}=323 \mathrm{~K}$ by HVID-COSMO-RS, COSMO-SAC and UNIFAC'93 model is illustrated in Fig. 1.

The temperature dependent parameters are required and the extrapolation at higher temperatures is used, as it was shown in previous papers [3-6]. An example is presented in Fig. 2 for the system: methanol + acetone, where the thick solid lines correspond to the correlation of parameters at low temperatures and the thin lines are the predictions at higher temperatures. Although differences are observed in both the low pressure values and the slopes of the lines, the extrapolations lead to satisfactory predictions of VLE at high pressures. The values of the constants in the temperature function (eq.12) obtained 
from UNIFAC'93 and both COSMO' type models are given in Table 3 for all studied systems. According to the procedure explained in the previous section, these functions were extrapolated to higher temperatures, in order to predict the vapor-liquid equilibria at higher temperatures and pressures using HVID mixing rules with the SRK equation of state coupled to the reduced UNIQUAC model. The average deviations resulting from both COSMO models and UNIFAC'93 can be observed in Table 4 for the studied binary systems.

Some examples show good predictions for the systems methanol + water, ethanol + water, acetone + water with both HVID-COSMO-RS and HVID-COSMO-SAC model (Fig. 3-5). A comparison of VLE predictions for acetone + water binary system at $\mathrm{T}=$ $473 \mathrm{~K}$ by the HVID-COSMO-RS, COSMO-SAC, UNIFAC'93 model is also given in Fig. 6, in order to make the fine details of the phase diagram clear. Differences of several bars are observed in the azeotropic pressure calculations with COSMO models. Due to the a priori predictive COSMO methods, which are based exclusively on uni-molecular quantum chemical calculations, such differences are explicable.

Binary parameters given in the Table 3 were used in VLE prediction of the ternary system acetone + methanol + water. Table 5 shows equilibrium calculation errors for the ternary VLE system. It is noted that both sets of parameters based on COSMO methods give good predictions in the ternary mixture. COSMO-SAC predictions are not as accurate as those from COSMO-RS. Surprisingly COSMO-RS predictions are slightly better than those obtained from UNIFAC'93. The comparison of VLE predicted pressures in the ternary system by HVID-COSMO-RS model with the experimental data is shown in the Fig. 7.

\section{Outlook}

A procedure previously proposed [3-6] is applied to predict vapor-liquid equilibria at high pressure using the HVID mixing rule model and the infinite dilution activity coefficients at low pressures from COSMO-RS, COSMO-SAC models.

COSMO-RS leads to good results and proved to be an alternative to the UNIFAC'93 model. Approximately the same average deviations resulting from both COSMO-RS and UNIFAC'93 can be observed in Table 4 for the studied binary systems, and the VLE prediction by COSMO-RS was slightly better for the ternary system acetone + methanol + water. COSMO-SAC predictions are not as accurate as those from COSMO-RS. This can be explained by the fact that several COSMO-RS default parameters, which have been used in COSMO-SAC, were not optimized in COSMO-SAC calculations [14].

Consequently, this work enlarges the field of application of COSMO-type methods to vapor-liquid equilibrium prediction at high pressures within the context of the HVID model. More extensive tests should be required, in order to validate the COSMO-type methods, but these are restricted by the availability of the quantum mechanical calculations for a larger number of chemical substances. The implementation of COSMO model is available at this time in the TURBOMOLE and DMOL3 quantum mechanical commercial software. 


\section{Acknowledgements}

The authors are greatful to Prof. S. I. Sandler and Dr. S. Lin (University of Delaware, USA) for sending the COSMO-SAC program V1.0. Two of the authors (D.C. and D.G.) are grateful to National Council of Scientific Research of Romania for financial support of this work.

\section{References}

[1] V. Feroiu and D. Geană, Rev. Roum. Chim., 34 (1989) 1329-1339.

[2] R. Stryjek and J.H. Vera, Fluid Phase Equilibria, 25 (1986) 279-290.

[3] V. Feroiu and D. Geană, Fluid Phase Equilibria, 120 (1996) 1-10.

[4] V. Feroiu and D. Geană, Rev. Roum. Chim., 42 (9) (1997) 863-871.

[5] D. Geană and V. Feroiu, Ind. Eng. Chem. Res., 37 (1998) 1173-1180.

[6] V. Feroiu and D. Geană, Rev. Roum. Chim., 44 (10) (1999) 941-948.

[7] J. Gmehling, J. Li and M. Schiller, Ind. Eng. Chem. Res., 32 (1993) 178-193.

[8] A. Klamt, J. Phys. Chem. 99 (1995) 2224-2235.

[9] A. Klamt and F. Eckert, Fluid Phase Equilibria, 172 (2000) 43-72.

[10] N. Tantipat, A. Klamt, I. Clausen and J. Anzelm, Ing. Abstr. Pap-Am. Chem. Soc 217 (1999) U671.

[11] A. Schäfer, A. Klamt, D. Sattel, J. C. W. Lohrenz and F. Eckert, Phys. Chem. Chem. Phys. 2 (2000) 2187-2193.

[12] A. Klamt, V. Jonas, T. Bürger and J. C. W. Lohrenz, J. Phys. Chem. A 102 (26) (1998) 5074-5085.

[13] R. Putnam, R. Taylor, A. Klamt, F. Eckert, and M. Schiller, Ind. Eng. Chem. Res., 42 (2003) 3635-3641.

[14] S. T. Lin and S. I. Sandler, Ind. Eng. Chem. Res., 41 (2002) 899-913.

[15] S. T. Lin and S. I. Sandler, AIChE J. 45 (1999) 2606-2618.

[16] O. Spuhl and W. Arlt, Ind. Eng. Chem. Res. 43 (2004) 852-861.

[17] C. Panayiotou, Ind. Eng. Chem. Res. 42 (2003) 1495-1507.

[18] G. Soave, Chem. Eng. Sci., 27 (1972) 1197-1203.

[19] P.M. Mathias and T.W. Copeman, Fluid Phase Equilibria, 13 (1983) 91-108.

[20] R.C. Reid, J. M. Prausnitz and B. E. Poling, "The properties of gases and liquids", McGraw-Hill, New York, 1988.

[21] S. Dahl, A. Fredenslund and P. Rasmussen, Ind. Eng. Chem. Res. 30 (1991)19361945.

[22] G. Soave, A. Bertucco, and L. Vecchiato, Ind. Eng. Chem. Res. 33 (1994) 975-980.

[23] D. Wong and S. Sandler, AIChE J., 38 (1992) 671-680.

[24] F. Barr-David and B.F.Dodge, J. Chem.Eng.Data 4 (1959) 107.

[25] J. Griswold and S. Y. Wong, Chem. Eng. Progr. Symp.Ser. 48 (1952) 18.

[26] J. M. Olewsky and I. F. Golubev, Tr. Gosudarst. Nauch.-Issled. I. Proekt. Inst. Azot. Prom. 6 (1956) 45.

[27] K. L. Butcher and M. S. Medani, J. Appl. Chem., 18 (1969) 100 
List of symbols

$\mathrm{a}, \mathrm{b}$
$\mathrm{C}_{1}, \mathrm{C}_{2}, \mathrm{C}_{3}$
$\mathrm{G}$
$\mathrm{q}$
$\mathrm{P}$
$\mathrm{R}$
$\mathrm{T}$
$\mathrm{V}$
$\mathrm{u}$
$\mathrm{x}$
$\mathrm{y}$
$\mathrm{Z}$

Greek Letters

$\sigma$

$\gamma$

$\tau, \theta$

Subscripts

$i, j$
$\mathrm{c}$
$\mathrm{r}$

Superscripts

$\mathrm{E}$

$\infty$

Abbreviations

COSMO-RS

COSMO-SAC

GCS

HVID

No Dpt

SRK

UNIQUAC

UNIFAC

VLE equation of state parameters

pure component temperature-dependence parameters

Gibbs free energy

UNIQUAC parameter

pressure

gas constant

temperature

molar volume

reduced UNIQUAC parameter, $\mathrm{K}$

mole fraction in the liquid phase

mole fraction in the vapor phase

compressibility factor

screening charge density

activity coefficient

UNIQUAC parameters

components

critical

reduced

excess

infinite dilution

conductor-like screening model for real solvents

segment activity coefficient model

group contribution solvation

Huron-Vidal mixing rule coupled with infinite dilution activity coefficients

number of data points

Soave-Redlich-Kwong equation of state

universal quasi chemical

UNIQUAC functional group activity coefficients

vapor-liquid equilibria 
Table 1 Infinite dilution activity coefficients at low temperature/pressure, calculated using COSMO-RS, SAC and UNIFAC models and infinite dilution activity coefficients at high pressure, calculated with eq. (9) for acetone (1) + water (2) system.

\begin{tabular}{llccccc}
\hline \multicolumn{1}{c}{ System } & \multicolumn{1}{c}{ Model } & $\mathbf{T} / \mathbf{K}$ & $\gamma_{1}^{\infty}(P \rightarrow 0)$ & $\gamma_{2}^{\infty}(P \rightarrow 0)$ & $\gamma_{1}^{\infty}(P \rightarrow \infty)$ & $\gamma_{2}^{\infty}(P \rightarrow \infty)$ \\
\hline \multirow{5}{*}{ COSMO-SAC } & \multirow{2}{*}{298} & 4.56 & 5.89 & 22.05 & 13.33 \\
& COSMO-RS & 4.40 & 4.87 & 21.20 & 10.70 \\
& UNIFAC'93 & & 8.04 & 7.07 & 41.03 & 16.46 \\
\cline { 2 - 7 } acetone(1)+ & COSMO-SAC & \multirow{2}{*}{303} & 4.70 & 5.87 & 22.95 & 13.42 \\
water(2) & COSMO-RS & 4.89 & 4.80 & 23.96 & 10.62 \\
& UNIFAC'93 & & 8.19 & 6.83 & 42.22 & 15.99 \\
\cline { 2 - 7 } & COSMO-SAC & \multirow{2}{*}{318} & 5.11 & 5.82 & 25.70 & 13.73 \\
& COSMO-RS & 6.41 & 4.57 & 33.03 & 10.32 \\
& UNIFAC'93 & & 8.57 & 6.18 & 45.55 & 14.73 \\
\cline { 2 - 7 } & COSMO-SAC & \multirow{2}{*}{333} & 5.49 & 5.74 & 28.49 & 14.00 \\
& COSMO-RS & 7.86 & 4.32 & 42.53 & 9.96 \\
& UNIFAC'93 & & 8.83 & 5.61 & 48.44 & 13.62 \\
\hline
\end{tabular}


Table 2 VLE results at low pressures for HVID, COSMO-RS, COSMO-SAC and UNIFAC model and SRK EOS

\begin{tabular}{|c|c|c|c|c|c|c|c|c|}
\hline \multirow[t]{2}{*}{ System } & \multirow[t]{2}{*}{$\mathbf{T}[\mathbf{K}]$} & \multirow[t]{2}{*}{$\begin{array}{l}\text { No } \\
\text { Dpt }\end{array}$} & \multicolumn{2}{|c|}{$\begin{array}{c}\text { HVID } \\
\text { COSMO-SAC }\end{array}$} & \multicolumn{2}{|c|}{$\begin{array}{c}\text { HVID } \\
\text { COSMO-RS }\end{array}$} & \multicolumn{2}{|c|}{$\begin{array}{c}\text { HVID } \\
\text { UNIFAC'93 }\end{array}$} \\
\hline & & & $\Delta P / P \times 100$ & $\Delta y \times 100$ & $\Delta P / P \times 100$ & $\Delta y \times 100$ & $\Delta P / P \times 100$ & $\Delta y \times 100$ \\
\hline \multirow{4}{*}{$\begin{array}{l}\text { ethanol(1) + } \\
\text { water(2) }\end{array}$} & 303.15 & 25 & 5.2 & 2.8 & 5.3 & 3.0 & 1.1 & 0.6 \\
\hline & 323.15 & 26 & 4.6 & 2.4 & 4.6 & 2.5 & 0.8 & 0.5 \\
\hline & 333.15 & 21 & 5.5 & 1.8 & 4.9 & 1.7 & 0.7 & 1.0 \\
\hline & 343.15 & 26 & 4.2 & 2.1 & 4.0 & 2.2 & 0.7 & 0.5 \\
\hline Average & & & 4.8 & 2.2 & 4.7 & 2.3 & 0.8 & 0.6 \\
\hline \multirow{6}{*}{$\begin{array}{l}\text { methanol (1)+ } \\
\text { benzene (2) }\end{array}$} & 298.15 & 9 & 11.7 & 5.3 & 4.0 & 2.6 & 2.4 & 1.9 \\
\hline & 308.15 & 9 & 9.8 & 4.9 & 6.8 & 4.7 & 2.1 & 1.7 \\
\hline & 311.65 & 7 & 8.3 & 3.2 & 4.3 & 2.3 & 3.1 & 1.7 \\
\hline & 313.15 & 14 & 10.6 & 6.1 & 1.3 & 1.4 & 1.5 & 1.2 \\
\hline & 318.15 & 25 & 6.6 & 4.5 & 8.6 & 4.4 & 1.9 & 1.9 \\
\hline & 328.15 & 7 & 7.5 & 3.2 & 3.7 & 1.6 & 3.3 & 1.1 \\
\hline Average & & & 9.0 & 4.5 & 4.7 & 2.8 & 2.3 & 1.5 \\
\hline \multirow{4}{*}{$\begin{array}{l}\text { 2-propanol(1)+ } \\
\text { water(2) }\end{array}$} & 303 & 18 & 9.5 & 5.9 & 8.1 & 5.4 & 1.4 & 1.5 \\
\hline & 308 & 9 & 8.9 & 5.5 & 6.6 & 4.2 & 2.9 & 0.7 \\
\hline & 318 & 18 & 9.0 & 6.1 & 7.4 & 5.3 & 1.2 & 2.3 \\
\hline & 333 & 18 & 8.8 & 6.1 & 6.6 & 4.8 & 1.9 & 3.0 \\
\hline Average & & & 9.0 & 5.9 & 7.1 & 4.9 & 1.8 & 1.8 \\
\hline \multirow{4}{*}{$\begin{array}{l}\text { acetone(1)+ } \\
\text { water(2) }\end{array}$} & 298 & 11 & 5.6 & 1.0 & 8.5 & 1.1 & 6.9 & 2.9 \\
\hline & 303 & 11 & 5.3 & 1.2 & 7.2 & 1.1 & 6.6 & 2.7 \\
\hline & 318 & 11 & 5.8 & 1.5 & 5.7 & 0.9 & 3.4 & 1.4 \\
\hline & 333 & 11 & 5.4 & 1.7 & 4.5 & 1.3 & 1.4 & 0.7 \\
\hline Average & & & 5.5 & 1.3 & 6.4 & 1.1 & 4.5 & 1.9 \\
\hline \multirow{5}{*}{$\begin{array}{l}\text { methanol(1) + } \\
\text { acetone(2) }\end{array}$} & 293 & 11 & 0.7 & 2.1 & 5.1 & 0.8 & 7.6 & 1.0 \\
\hline & 303 & 7 & 6.1 & 2.6 & 2.3 & 2.5 & 0.9 & 2.5 \\
\hline & 313 & 8 & 6.7 & 2.8 & 2.7 & 2.3 & 0.5 & 2.0 \\
\hline & 318 & 11 & 4.7 & 2.4 & 2.0 & 1.3 & 0.9 & 0.5 \\
\hline & 328 & 28 & 5.8 & 2.6 & 3.4 & 1.6 & 0.8 & 0.3 \\
\hline Average & & & 4.8 & 2.5 & 3.1 & 1.7 & 2.1 & 1.2 \\
\hline \multirow{6}{*}{$\begin{array}{l}\text { methanol(1) + } \\
\text { water(2) }\end{array}$} & 289 & 14 & 2.3 & 0.8 & 3.9 & 1.1 & 0.8 & 0.3 \\
\hline & 308 & 14 & 2.6 & 1.3 & 3.7 & 1.4 & 1.8 & 1.0 \\
\hline & 313 & 10 & 2.0 & 0.9 & 4.0 & 1.4 & 0.9 & 0.3 \\
\hline & 323 & 13 & 1.8 & 0.8 & 2.9 & 1.1 & 0.7 & 0.3 \\
\hline & 333 & 12 & 2.1 & 1.5 & 3.3 & 1.9 & 0.9 & 0.8 \\
\hline & 338 & 12 & 1.5 & 0.5 & 2.3 & 0.8 & 0.7 & 0.2 \\
\hline Average & & & 2.0 & 0.9 & 3.3 & 1.2 & 0.9 & 0.4 \\
\hline
\end{tabular}


Table 3 The constants of the temperature function (eq.12) obtained from COSMO-RS, COSMO-SAC and UNIFAC'93 activity coefficients at infinite dilution at low temperatures.

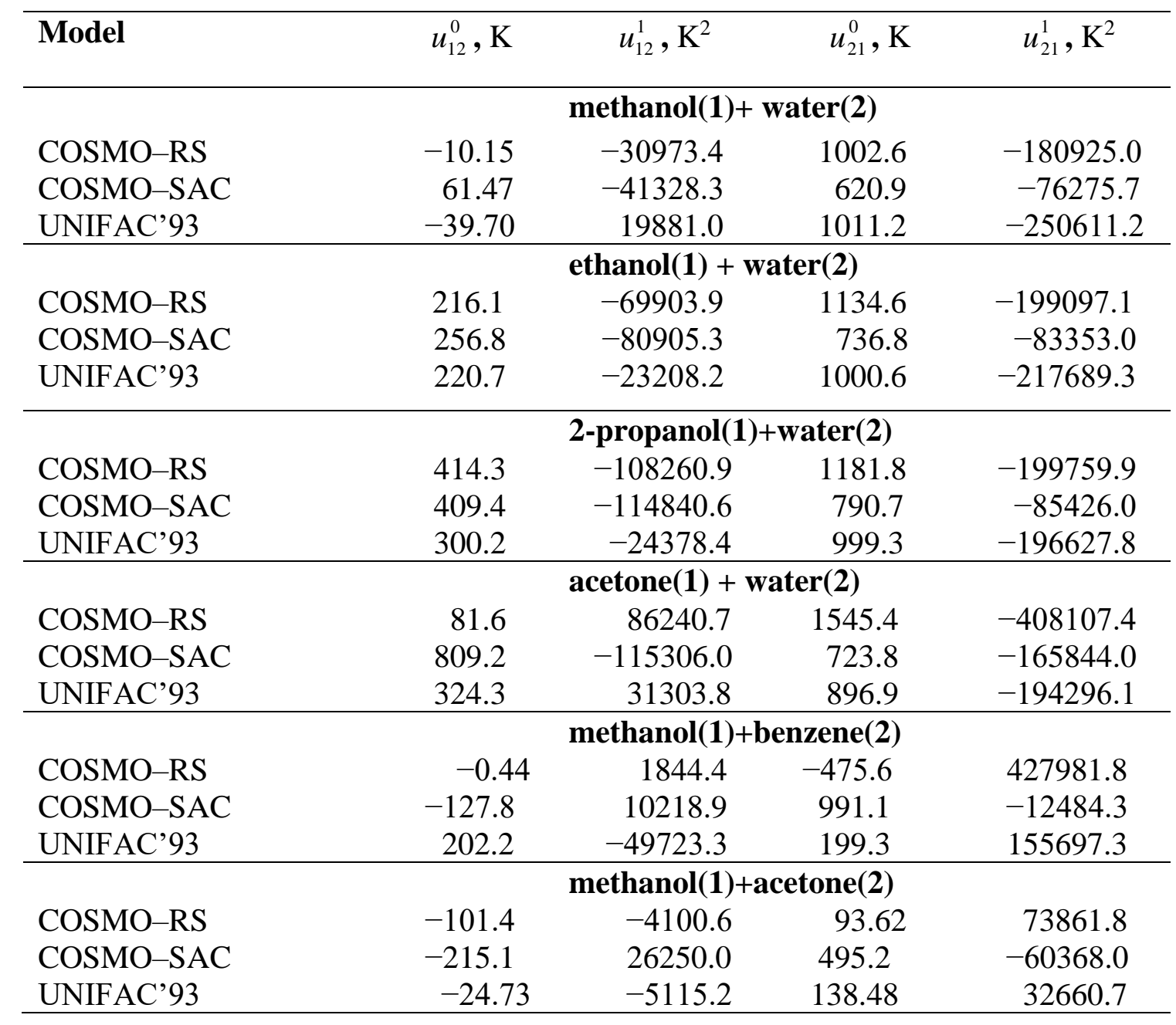


Table 4 Prediction of VLE at high pressures by HVID mixing rules with reduced UNIQUAC model and SRK EOS, from COSMO-RS, COSMO-SAC and UNIFAC'93 activity coefficients at infinite dilution

\begin{tabular}{|c|c|c|c|c|c|c|c|c|c|c|}
\hline System & Lit & $\mathbf{T}[\mathbf{K}]$ & $\mathbf{P}[$ bar $]$ & $\begin{array}{l}\text { No. } \\
\text { Dpt. }\end{array}$ & $\begin{array}{l}\mathrm{COS} \\
\Delta \mathrm{P} / \mathrm{P} \\
\times 100\end{array}$ & $\begin{array}{c}\text { MO-RS } \\
\Delta y \\
\times 100\end{array}$ & $\begin{array}{r}\mathrm{HV} \\
\mathrm{COSM} \\
\Delta \mathrm{P} / \mathrm{P} \\
\times 100\end{array}$ & $\begin{array}{l}\text { ID } \\
\text { O-SAC } \\
\begin{array}{c}\Delta y \\
\times 100\end{array}\end{array}$ & \multicolumn{2}{|c|}{$\begin{array}{l}\text { UNIFAC'93 } \\
\Delta \mathrm{P} / \mathbf{P} \quad \Delta y \\
\times \mathbf{1 0 0} \times \mathbf{1 0 0}\end{array}$} \\
\hline \multirow{6}{*}{$\begin{array}{l}\text { methanol(1)+ } \\
\text { water(2) }\end{array}$} & [25] & 373 & $1.0-3.4$ & 16 & 1.0 & 1.1 & 1.1 & 0.8 & 2.0 & 1.1 \\
\hline & [25] & 423 & $5.0-13.7$ & 11 & 1.1 & 0.9 & 1.8 & 1.2 & 1.0 & 1.1 \\
\hline & {$[26]$} & 443 & $6.7-20.9$ & 35 & 5.4 & 3.0 & 5.0 & 3.3 & 4.5 & 3.5 \\
\hline & [25] & 473 & $15.5-38.3$ & 17 & 3.6 & 1.7 & 4.7 & 1.9 & 2.9 & 2.2 \\
\hline & [26] & 508 & $30.6-70.6$ & 18 & 3.8 & 1.7 & 2.2 & 1.8 & 4.5 & 1.9 \\
\hline & [25] & 523 & $46.9-68.5$ & 6 & 2.6 & 1.3 & 0.6 & 0.5 & 2.5 & 1.3 \\
\hline \multicolumn{5}{|l|}{ Averages } & 2.9 & 1.6 & 2.5 & 1.6 & 2.9 & 1.8 \\
\hline \multirow{5}{*}{$\begin{array}{l}\text { ethanol(1)+ } \\
\text { water(2) }\end{array}$} & [24] & 423 & $5.6-9.9$ & 17 & 1.6 & 1.3 & 3.1 & 2.0 & 1.7 & 0.5 \\
\hline & [24] & 473 & $17.9-29.5$ & 17 & 1.6 & 1.1 & 2.9 & 1.2 & 1.6 & 0.5 \\
\hline & [24] & 523 & $40.8-71.7$ & 18 & 1.9 & 0.8 & 2.4 & 0.8 & 1.8 & 0.2 \\
\hline & [24] & 548 & $61.3-100.4$ & 13 & 2.1 & 1.4 & 2.4 & 0.9 & 1.4 & 0.6 \\
\hline & [24] & 573 & $88.5-128.9$ & 9 & 2.1 & 1.0 & 2.2 & 1.2 & 0.3 & 0.6 \\
\hline \multicolumn{5}{|l|}{ Averages } & 1.8 & 1.1 & 2.6 & 1.2 & 1.4 & 0.5 \\
\hline \multirow{5}{*}{$\begin{array}{l}\text { 2-propanol(1) } \\
+ \text { water(2) }\end{array}$} & [24] & 423 & $5.2-8.6$ & 18 & 2.6 & 1.8 & 6.7 & 4.0 & 2.5 & 2.1 \\
\hline & [24] & 473 & $18.5-26.1$ & 18 & 1.8 & 1.3 & 6.3 & 3.0 & 3.0 & 1.5 \\
\hline & [24] & 523 & $43.1-64.9$ & 16 & 1.6 & 1.7 & 5.6 & 3.4 & 2.5 & 1.8 \\
\hline & [24] & 548 & $69.0-92.9$ & 18 & 0.9 & 2.2 & 6.1 & 2.8 & 2.9 & 7.6 \\
\hline & [24] & 573 & $88.9-123.5$ & 6 & 1.2 & 4.6 & 5.0 & 5.7 & 2.9 & 6.2 \\
\hline \multicolumn{5}{|l|}{ Averages } & 1.6 & 2.3 & 5.9 & 3.7 & 2.7 & 3.8 \\
\hline \multirow{4}{*}{$\begin{array}{l}\text { acetone(1)+ } \\
\text { water(2) }\end{array}$} & [25] & 373 & $1.1-3.7$ & 22 & 2.6 & 0.8 & 5.0 & 2.5 & 2.4 & 0.9 \\
\hline & [25] & 423 & $6.7-11.7$ & 14 & 2.2 & 0.3 & 3.0 & 2.3 & 1.2 & 1.2 \\
\hline & [25] & 473 & $16.0-27.9$ & 25 & 1.9 & 0.7 & 3.9 & 2.0 & 1.8 & 1.1 \\
\hline & [25] & 523 & $40.4-67.6$ & 14 & 2.2 & 1.1 & 1.6 & 1.7 & 2.8 & 0.9 \\
\hline \multicolumn{5}{|l|}{ Averages } & 2.3 & 0.7 & 3.4 & 2.1 & 2.1 & 1.0 \\
\hline \multirow{6}{*}{$\begin{array}{l}\text { methanol(1)+ } \\
\text { benzene }(2)\end{array}$} & [27] & 393 & $2.9-6.4$ & 12 & 2.6 & 1.1 & 4.8 & 2.3 & 2.2 & 0.8 \\
\hline & [27] & 413 & $4.7-10.8$ & 12 & 3.6 & 1.6 & 4.7 & 2.8 & 2.3 & 0.9 \\
\hline & [27] & 433 & $7.1-17.3$ & 12 & 4.4 & 2.1 & 4.3 & 2.6 & 2.3 & 0.7 \\
\hline & [27] & 453 & $10.2-26.7$ & 12 & 4.0 & 1.9 & 4.2 & 2.6 & 3.5 & 1.2 \\
\hline & [27] & 473 & $14.2-39.5$ & 12 & 5.1 & 2.7 & 3.9 & 2.6 & 3.0 & 1.4 \\
\hline & [27] & 493 & $19.4-56.8$ & 12 & 4.2 & 3.0 & 3.6 & 3.6 & 3.2 & 2.7 \\
\hline \multicolumn{5}{|l|}{ Averages } & 4.0 & 2.1 & 4.3 & 2.8 & 2.8 & 3.7 \\
\hline \multirow{5}{*}{$\begin{array}{l}\text { methanol(1)+ } \\
\text { acetone(2) }\end{array}$} & [25] & 373 & $3.5-4.1$ & 14 & 1.2 & 1.5 & 1.8 & 1.9 & 2.0 & 1.2 \\
\hline & [25] & 385 & $5.2-5.6$ & 10 & 2.7 & - & 3.2 & - & 0.5 & - \\
\hline & [25] & 397 & $6.6-7.8$ & 11 & 2.2 & 0.8 & 2.4 & 0.9 & 0.6 & 0.8 \\
\hline & [25] & 423 & $11.7-14.2$ & 15 & 2.5 & 2.1 & 2.1 & 2.1 & 0.7 & 1.7 \\
\hline & [25] & 473 & $29.5-39.9$ & 10 & 2.7 & 3.2 & 2.1 & 3.1 & 2.7 & 3.1 \\
\hline \multicolumn{5}{|l|}{ Averages } & 2.3 & 1.9 & 2.3 & 2.0 & 1.3 & 1.7 \\
\hline
\end{tabular}


Table 5 High pressures VLE prediction for the ternary system acetone-methanol-water by HVID mixing rules with reduced UNIQUAC model and SRK EOS, from COSMORS, COSMO-SAC and UNIFAC'93 activity coefficients at infinite dilution. Experimental data of Griswold and Wong [25]

\begin{tabular}{|c|c|c|c|c|c|c|c|c|c|c|c|}
\hline \multirow[t]{2}{*}{$\mathbf{T},[\mathbf{K}]$} & \multirow[t]{2}{*}{ P, bar } & \multirow{2}{*}{$\begin{array}{l}\text { No } \\
\text { Dpt }\end{array}$} & \multicolumn{3}{|c|}{ COSMO-RS } & \multicolumn{3}{|c|}{ COSMO-SAC } & \multicolumn{3}{|c|}{ UNIFAC' ${ }^{93}$} \\
\hline & & & $\begin{array}{l}\mathbf{\Delta P} / \mathbf{P} \\
\mathrm{x} 100\end{array}$ & $\begin{array}{l}\Delta \mathbf{\Delta y} 1 \\
\mathrm{x} 100\end{array}$ & $\begin{array}{l}\Delta \mathbf{y}_{2} \\
\mathrm{x} 100\end{array}$ & $\begin{array}{l}\mathbf{\Delta P} / \mathbf{P} \\
\mathrm{x} 100\end{array}$ & $\begin{array}{l}\Delta \mathbf{\Delta y \mathbf { 1 }} \\
\mathrm{x} 100\end{array}$ & $\begin{array}{l}\Delta \mathbf{y}_{2} \\
\mathrm{x} 100\end{array}$ & $\begin{array}{l}\Delta \mathbf{\Delta P} / \mathbf{P} \\
\mathrm{x} 100\end{array}$ & $\begin{array}{l}\Delta \mathbf{y} 1 \\
\text { x100 }\end{array}$ & $\begin{array}{l}\Delta \mathbf{\Delta y 2} \\
\mathrm{x} 100\end{array}$ \\
\hline 373.15 & $1.2-3.9$ & 50 & 2.5 & 2.5 & 3.0 & 4.3 & 3.6 & 3.4 & 3.2 & 2.3 & 3.0 \\
\hline 523.15 & $47-82$ & 56 & 1.5 & 0.7 & 1.6 & 2.2 & 1.0 & 1.5 & 2.3 & 0.8 & 3.0 \\
\hline
\end{tabular}


Fig.1 VLE predictions for methanol (1) + water (2) binary system at $\mathrm{T}=323 \mathrm{~K}$ by HVID-COSMO-RS (-), COSMO-SAC (---), UNIFAC'93(-) model. Experimental data $(0, \diamond)[25]$.

Fig.2 Methanol (1) + acetone (2) binary system. The temperature dependence of reduced UNIQUAC (HVID) parameters obtained from COSMO-RS ( $\square$ ), COSMO-SAC (०), and UNIFAC'93 $(\Delta)$ activity coefficients at infinite dilution. The thick solid lines correspond to the correlation of parameters at low temperatures and the thin lines are the predictions at higher temperatures.

Fig. 3 VLE predictions for methanol (1) + water (2) system by HVID-COSMO-RS (-), COSMO-SAC (---) model. Experimental data $(0, \diamond)[25,26]$.

Fig. 4 VLE predictions for ethanol (1) + water (2) system with HVID-COSMO-RS (-), COSMO-SAC (---) model. Experimental data $(\mathrm{O}, \diamond)[24]$.

Fig. 5 VLE predictions for acetone (1) + water (2) system with HVID-COSMO-RS (-), COSMO-SAC (---) model. Experimental data $(\mathrm{O}, \diamond)[25]$.

Fig. 6 Comparison of VLE predictions for acetone (1) + water (2) binary system at $\mathrm{T}=$ $473 \mathrm{~K}$ by HVID-COSMO-RS (-), COSMO-SAC (---), UNIFAC'93(-) model. Experimental data $(0, \diamond)[25]$.

Fig. 7 Comparison of VLE predictions for acetone(1) + methanol(2) + water(3) ternary system at T $=523 \mathrm{~K}$ by HVID-COSMO-RS model (O) with experimental data ( $)$ [25]. 


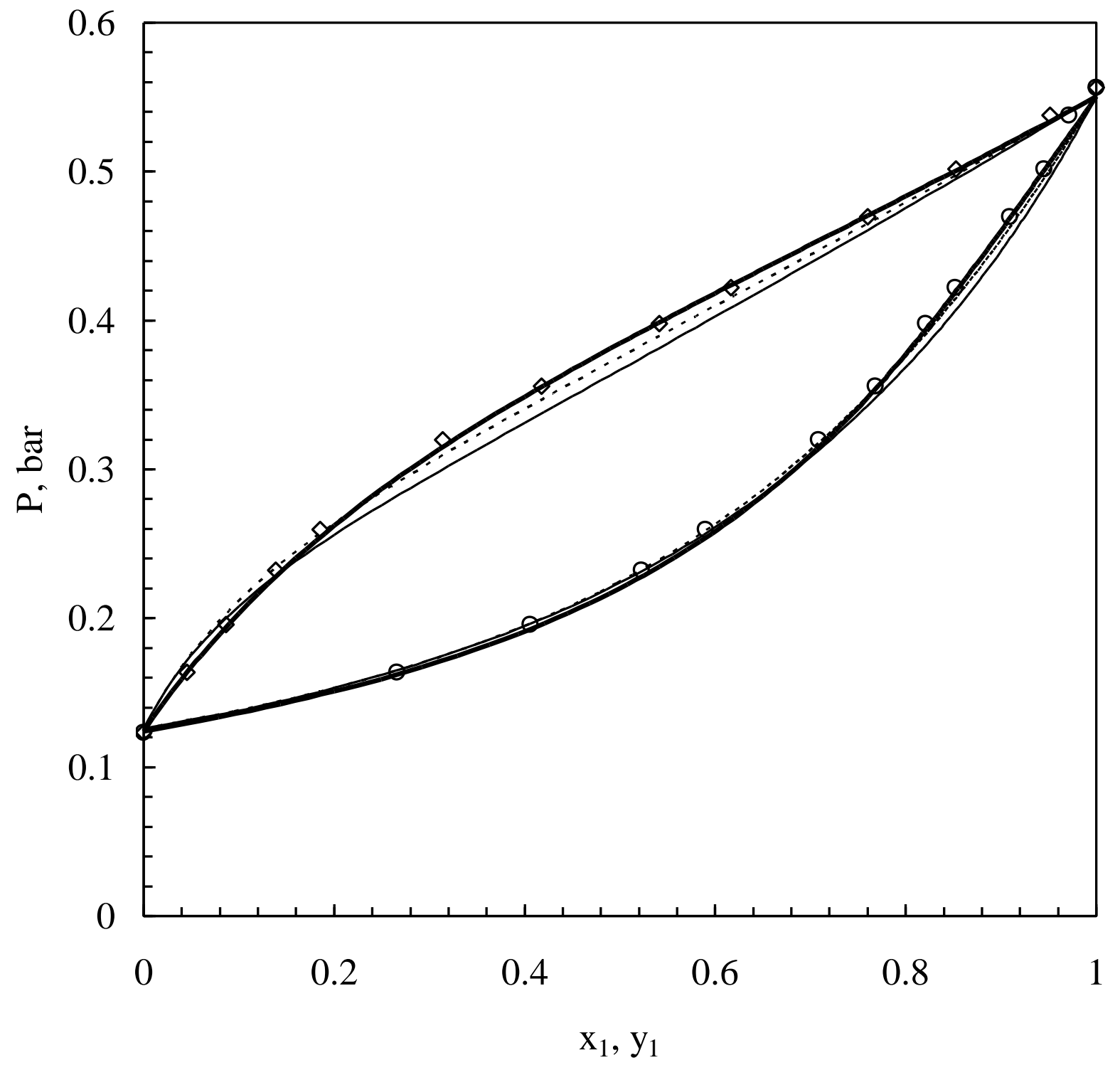

Fig. 1 


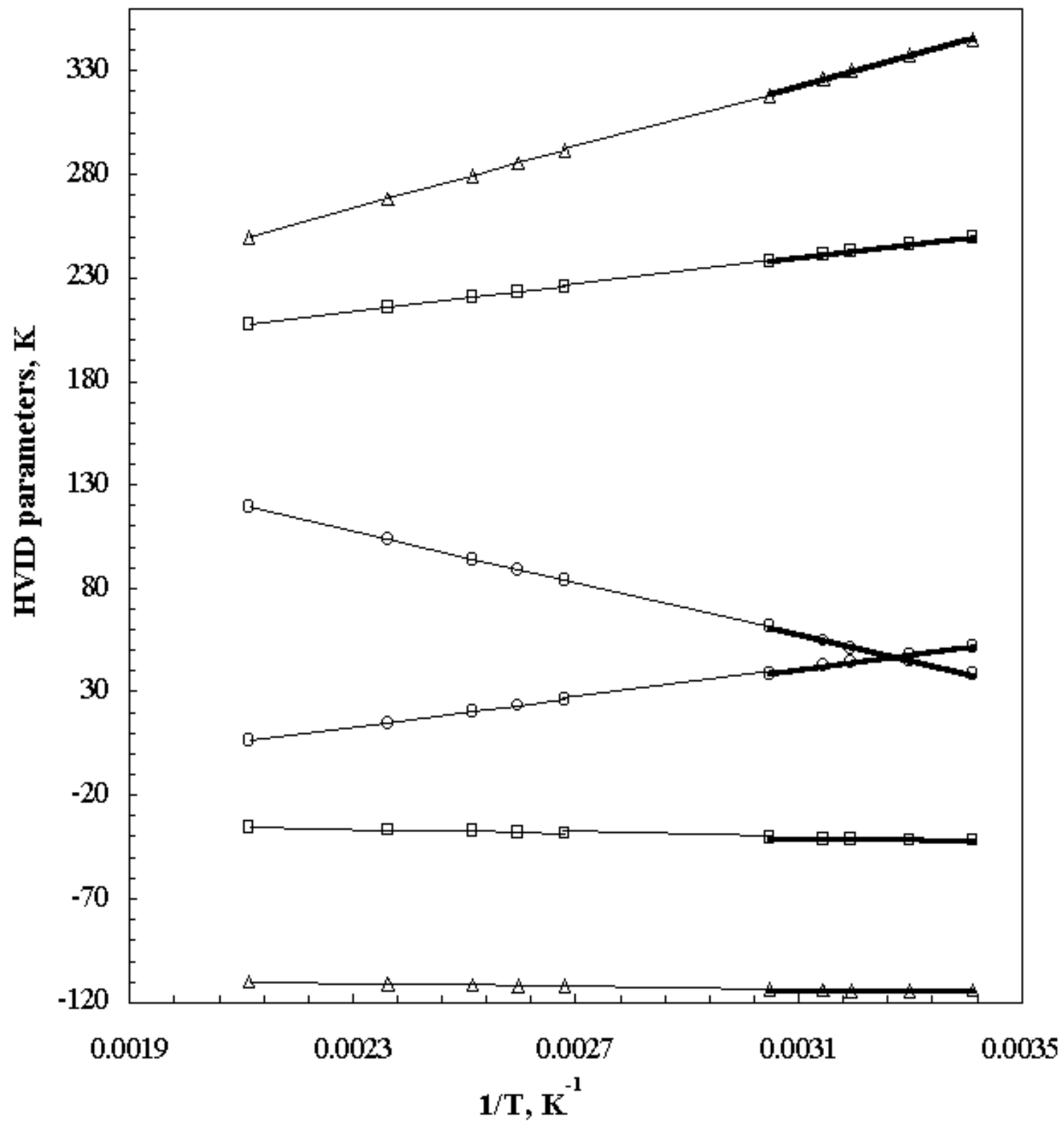

Fig. 2 


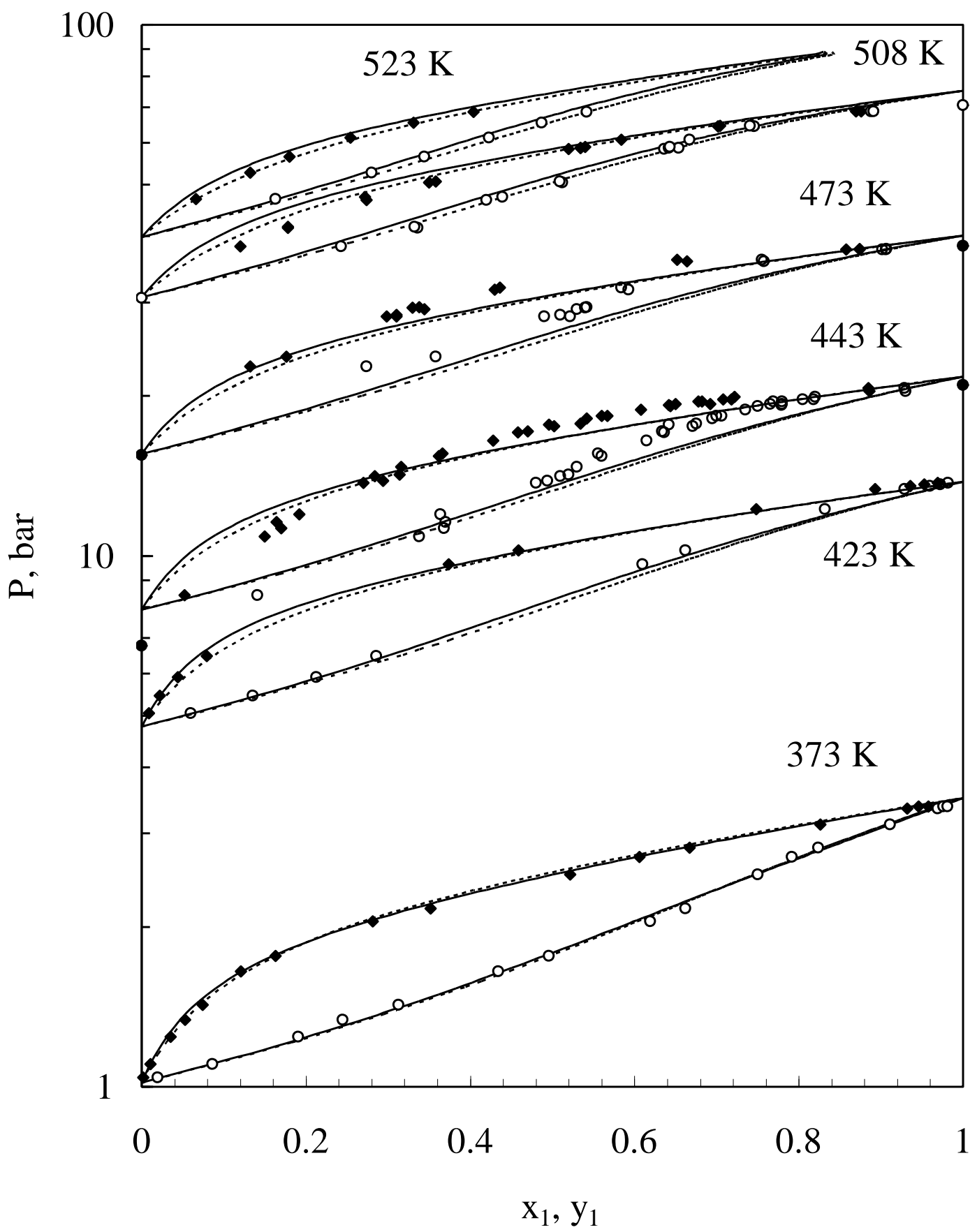

Fig. 3 


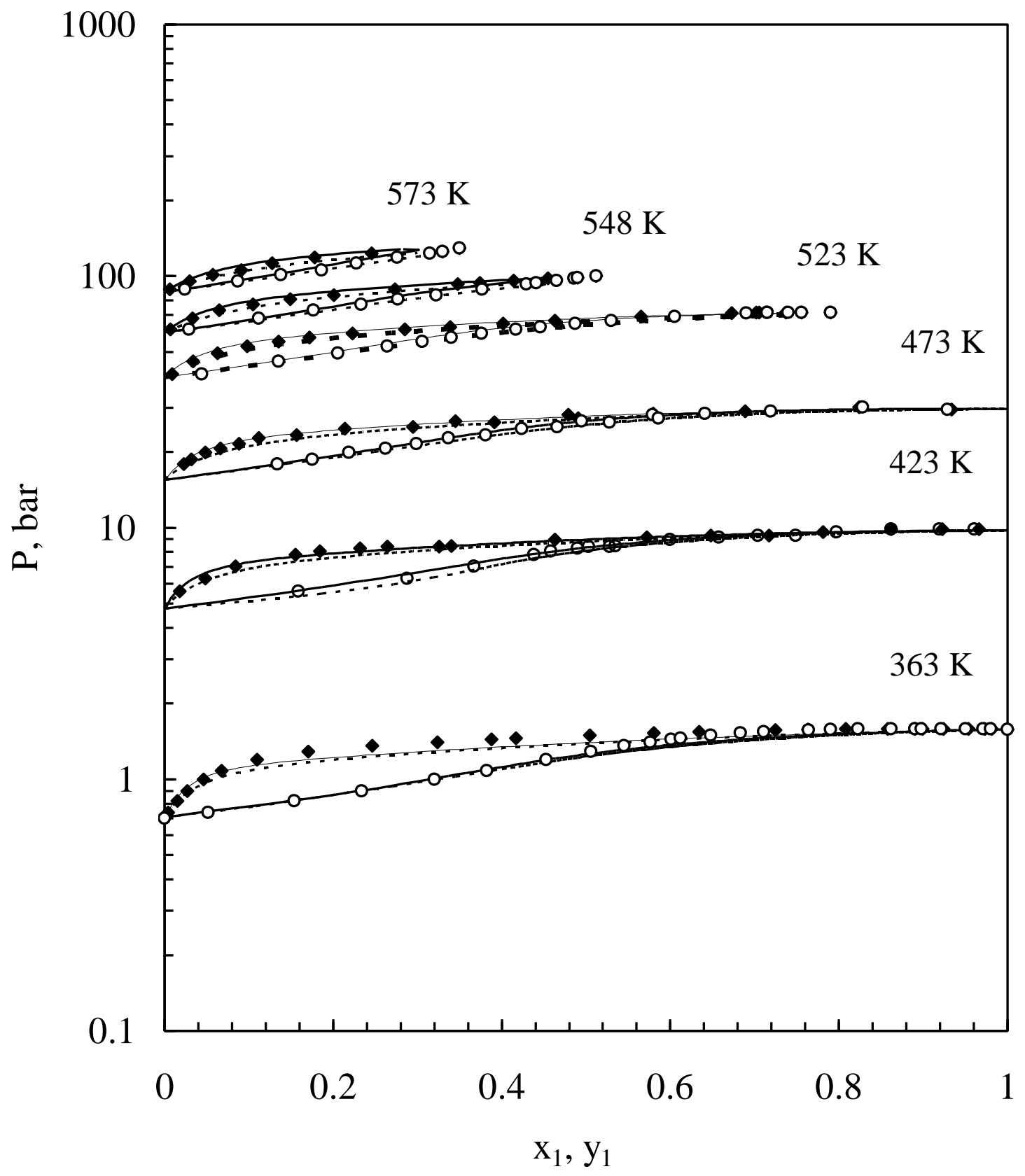

Fig. 4 


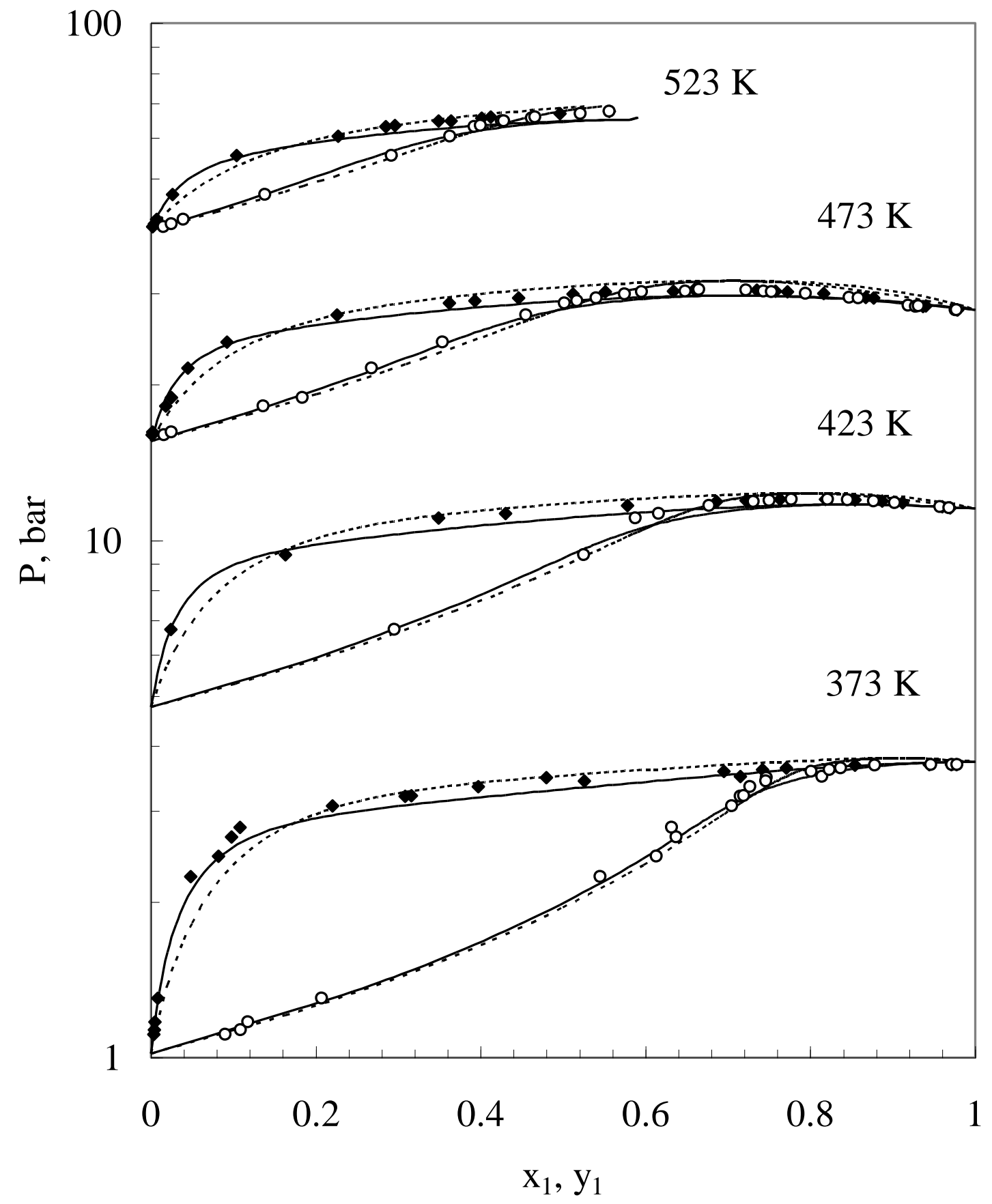

Fig. 5 


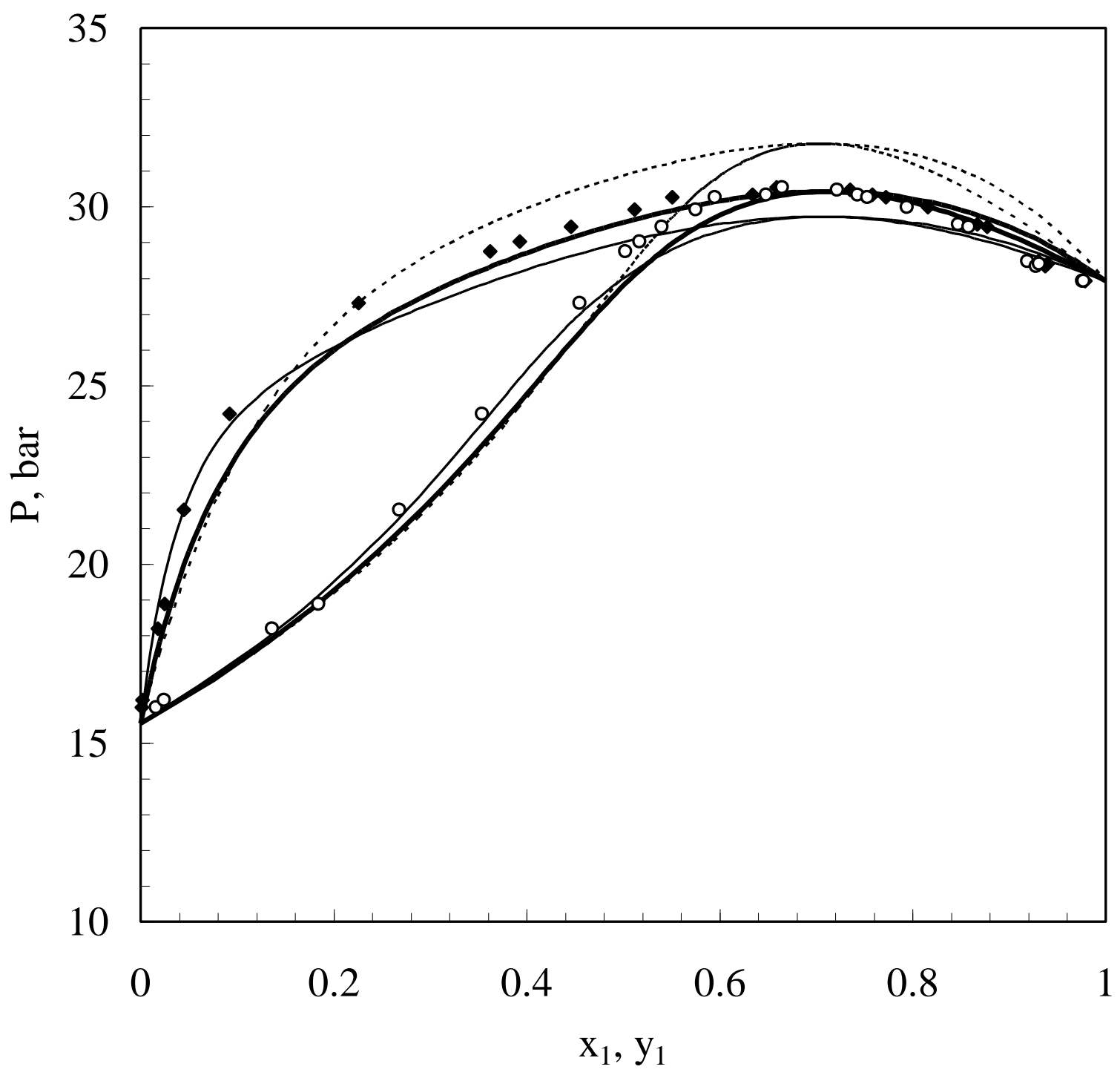

Fig.6 


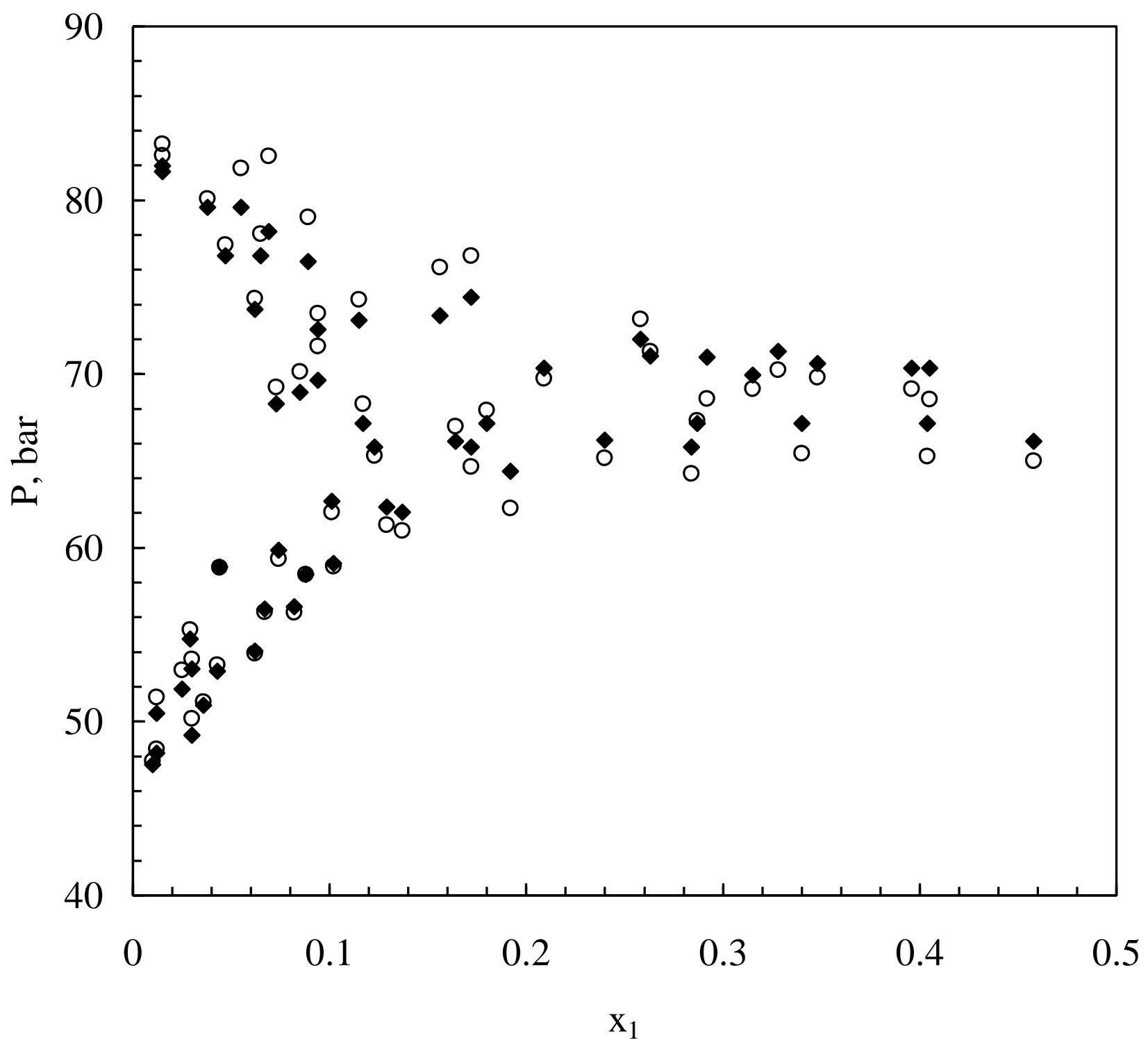

Fig. 7 